\title{
Aloe Vera y la Descontaminación Electromagnética
}

\section{Aloe Vera and Electromagnetic Decontamination}

\author{
Demetrio Soto-Carbajal \\ Universidad Nacional de Huancavelica, Huancavelica, Perú \\ E-mail: demetrio.soto@unh.edu.pe ORCID: https://orcid.org/0000-0001-8639-2954 \\ Lizangela Aurelia Hinojosa-Yzarra \\ Universidad Nacional Autónoma de Huanta, Ayacucho, Perú \\ E-mail: 1912820118@unah.edu.pe ORCID: https://orcid.org/0000-0001-9567-0303
}

Recepción: 15/06/2021 Aceptación: 30/06/2021 Publicación: 30/07/2021

\section{Resumen}

La población mundial sufre de alteraciones al corazón y el sistema respiratorio, generados por las ondas electromagnéticas provenientes de los ordenadores, celulares y microondas, ocasionando una serie de infartos de miocardio que dan lugar a la disminución de su masa muscular, de tal manera que la onda se despolariza lentamente por los ventrículos del corazón. El objetivo del presente estudio fue demostrar la influencia del Aloe Vera (sábila) en la descontaminación de los efectos electromagnéticos en los seres vivos, para lo cual se desarrolló un estudio sistemático de artículos científicos publicados en revistas científicas como Guillermo de Ockham, Dilemas Contemporáneos, Airalia, entre otras, ubicadas en bases de datos como Scopus, SciELO, Web of Science, Latindex y ScienceDirect. Entre los resultados se destaca la confirmación de la estrecha relación entre radiaciones electromagnéticas y el cáncer, vislumbrándose la solución mediante un análisis de absorción ultravioleta (UV) y pruebas de citotoxicidad del Aloe vera, ante el daño oxidativo celular y la genotoxicidad producida por las partículas nanométricas inorgánicas sobre células dérmicas. Se concluyó que las radiaciones entre $30 \mathrm{kHz}$ y $300 \mathrm{MHz}$ y las microondas entre $300 \mathrm{MHz}$ y $300 \mathrm{GHz}$, alteran molecularmente el sistema nervioso; y para descontaminar los efectos de las ondas electromagnéticas, el gel del Aloe vera mostró actividades fisiológicas y biológicas como agente antiviral y protector ante la radiación UV.

\section{Palabras Clave}

Contaminación electromagnética, Aloe vera, ultravioleta. 


\begin{abstract}
The world population suffers from alterations to the heart and respiratory system, generated by electromagnetic waves from computers, cell phones and microwaves, causing a series of myocardial infarctions that lead to a decrease in muscle mass, in such a way that the wave depolarizes slowly through the ventricles of the heart. The aim was to demonstrate the influence of Aloe Vera (sábila) in the decontamination of electromagnetic effects in living beings, for which a systematic study of scientific articles published in scientific journals such as Guillermo de Ockham, Contemporary Dilemmas, was developed, Airalia, among others, located in databases such as Scopus, SciELO, Web of Science, Latindex and ScienceDirect. Among the results, the confirmation of the close relationship between electromagnetic radiation and cancer stands out, the solution being glimpsed through an ultraviolet (UV) absorption analysis. and cytotoxicity tests of Aloe vera, in the face of cellular oxidative damage and genotoxicity produced by inorganic nanometric particles on dermal cells. It was concluded that radiation between $30 \mathrm{kHz}$ and $300 \mathrm{MHz}$ and microwaves between $300 \mathrm{MHz}$ and $300 \mathrm{GHz}$, molecularly alter the nervous system; and to decontaminate the effects of electromagnetic waves, Aloe vera showed physiological and biological activities as an antiviral and protective agent against UV radiation.
\end{abstract}

\title{
Keywords
}

Electromagnetic pollution, Aloe vera, ultraviolet.

\section{Resumo}

A população mundial sofre alterações no sistema cardíaco e respiratório, geradas por ondas eletromagnéticas de computadores, telefones celulares e microondas, causando uma série de infartos do miocárdio que levam à diminuição da massa muscular, de forma que a onda se despolariza lentamente através os ventrículos do coração. O objetivo deste estudo foi demonstrar a influência do Aloe Vera (sábila) na descontaminação dos efeitos eletromagnéticos em seres vivos, para o qual foi desenvolvido um estudo sistemático de artigos científicos publicados em periódicos científicos como Guillermo de Ockham, Contemporary Dilemmas. Airalia, entre outras, localizada em bases de dados como Scopus, SciELO, Web of Science, Latindex e ScienceDirect. Entre os resultados, destaca-se a confirmação da estreita relação entre radiação eletromagnética e câncer, sendo a solução vislumbrada por meio de uma análise de absorção ultravioleta (UV) e testes de citotoxicidade de Aloe vera, frente ao dano oxidativo celular e genotoxicidade produzida por partículas nanométricas inorgânicas nas células dérmicas. Concluiu-se que radiações entre $30 \mathrm{kHz}$ e $300 \mathrm{MHz}$ e microondas entre $300 \mathrm{MHz}$ e $300 \mathrm{GHz}$ alteram molecularmente o sistema nervoso; e para descontaminar os efeitos das ondas eletromagnéticas, o gel de Aloe vera apresentou atividades fisiológicas e biológicas como agente antiviral e protetor contra a radiação ultravioleta.

\section{Palavras-chave}

Poluição eletromagnética, Aloe vera, ultravioleta. 


\section{Introducción}

Actualmente la contaminación electromagnética es uno de los peligros más mortificantes y silenciosos para el ser humano, de tal manera que las tecnologías 1G, 2G, 3G, 4G y 5G, creadas por el mismo hombre, avanzaron a pasos agigantados y está invadiéndonos a lo largo y ancho de nuestro planeta, razón por la cual en un futuro no muy lejano este veneno consumirá al ser vivo transformándolo en seres mutantes (Catalán, 2020). La salud humana y de todos los seres vivos corren peligro si este tipo de contaminación no se mitiga adecuadamente con leyes y normas jurídicas de aplicación severa. Las autoridades locales, regionales y nacionales o el mismo Estado, tienen la responsabilidad de controlar y evitar este tipo de contaminación a través de sus organismos gubernamentales, ministerios y otros, de tal manera que la disminución de este problema se constituya en un alivio y respiro para nuestro medio y, por ende, al bienestar del ser humano.

Como es de conocimiento, los problemas de la CEM implican trabajar en conjunto para planificar acciones de índole personal o grupal, gestando así alternativas de solución para resolver esta compleja misión que nos aqueja en el mundo entero. Visto esta problemática desde diferentes ángulos, encontramos entre diversas soluciones que permiten contrarrestar la CEM, la utilización de la planta Aloe vera (sábila), que mediante su proceso de fotosíntesis disminuye de alguna manera los efectos nocivos para la salud humana, gracias a un componente denominado gel que se constituye en dos fases: un sistema coloidal de fase continua o sólida y la fase dispersa o líquida, estos extractos vegetales son ricos en flavonoides y otras sustancias de acción anti irritantes y antiinflamatorias, constituyéndose así el Aloe vera en un vehículo seguro y compatible para acarrear esta problemática de la CEM (Cortes et al., 2020; Ledesma, 2019 y Vargas et al., 2020). El presente estudio realiza una revisión completa sobre la influencia de Aloe vera en la disminución de la CEM para analizar el estado actual e identificar la variedad de efectos causados por la emisión electromagnética con el objetivo de alentar a las autoridades, 
investigadores y la población en general a trabajar en la mejora de la salud humana, a través de sugerencias y recomendaciones.

\section{Descontaminación electromagnética}

\subsection{Contaminación electromagnética y sus efectos en la salud}

El uso masivo de dispositivos móviles, el incremento de fuentes generadoras de los campos electromagnéticos en los hogares y el inicio de la instalación de antenas para la tecnología $5 \mathrm{G}$ se ha generado preocupación social y las demandas de sectores implicados ante la necesidad de una norma que permita proteger a los ciudadanos de los posibles efectos a la salud que los campos electromagnéticos (CEM) pueden ocasionar. Como fuentes generadoras de radiaciones no ionizantes se encuentran las líneas de transmisión eléctrica y las estaciones de transformación $(50-60 \mathrm{~Hz})$, las fuentes conmutadas, las estaciones de radiodifusión de amplitud modulada (525- $1735 \mathrm{kHz})$, las estaciones de radiodifusión de frecuencia modulada (88-108 MHz), las estaciones de televisión (VHF y UHF) y los sistemas de comunicaciones móviles por celdas (800 y 1900 MHz), entre otras tecnologías empleadas en la transmisión eléctrica, las telecomunicaciones, en equipos industriales, médicos y electrodomésticos. Algunos estudios alertan sobre los efectos a la salud como el cáncer y cambios en el comportamiento de las personas. (Sampedro et al., 2020).

Las empresas de telefonía móvil poseen torres de antenas de alta potencia, las cuales trabajan a frecuencias de 850 y 1900 MHZ, y también las celdas de GSM que trabajan a menores frecuencias, las torres están ubicadas estratégicamente para lograr que las señales sean captadas por la mayor cantidad de equipos móviles (celulares) posibles. Las mediciones se realizaron en dos puntos de la ciudad donde las torres son de mayor potencia, y las distancias tomadas al nivel del suelo varían a partir de los 2 hasta los $200 \mathrm{~m}$ de distancia con respecto a las torres de antenas.

La medida más efectiva contra las radiaciones electromagnéticas es alejarse de las fuentes, mediante procedimientos sencillos, ejemplo, que el uso de celulares sea solamente para llamadas de corta duración, utilizar auriculares para mantener la antena alejada de la 
cabeza y que los niños no usen el equipo siendo ellos los más vulnerables dado que su sistema nervioso se encuentra en pleno desarrollo (Rolín et al., 2014).

Los contaminantes atmosféricos provienen de los vehículos propulsados por combustible, las actividades comerciales y la erosión del suelo, la contaminación atmosférica entrega evidencia de los problemas que estas pueden causar en poblaciones urbanas de las grandes metrópolis, donde se destaca a México entre otras capitales del mundo. La investigación puntualiza padecimientos físicos como bronquitis, neumonía y enfermedades pulmonares como efecto de dichos contaminantes. Las partículas contaminantes gruesas son las que se encuentran entre el rango del diámetro aerodinámico de los 2,5 (PM2,5) y los 10 micrómetros - medida que corresponde a la milésima parte de un milímetro- (PM10); las finas de entre $0,1(P M 0,1)$ y 2,5 (PM2,5), y las ultrafinas son menores de 0,1 (<PM0,1), que se consideran las más toxicas, puesto que pueden penetrar hasta cubrir una mayor área alveolar en el organismo de los humanos. Las partículas PM2,5 se consideran respirables al $100 \%$ y al formar parte de las partículas finas, la evidencia las ubica como de mayor riesgo para enfermedades respiratorias. (Leal, J.-lga. 2019).

La tecnología de quinta generación (5G) utiliza ondas de frecuencia más alta que las redes previas de telefonía celular. Estas ondas viajan distancias más cortas y por eso se requieren más antenas de transmisión y son más bajas. Si bien emplea campos electromagnéticos de mayor frecuencia, no se salen del espectro de radiación no ionizante (Tutivén, 2015). Los únicos efectos biológicos probados de la radiación no ionizante son "térmicos", es decir, el calentamiento de la materia biológica por absorción de la energía radiada. Aunque este calentamiento debe ser mínimo o despreciable a la intensidad legal.

Los daños causados por los campos electromagnéticos han sido declarados por la Organización Mundial de la Salud (OMS), como un problema de salud mundial. Siendo los niños y los trabajadores del sector eléctrico los más estudiados por los científicos de todo el mundo. Debido a que se ha comprobado el daño que ocasionan la exposición prolongada a este tipo de radiación y a la ausencia de una normativa acerca de este tema en nuestro país, es necesario comprobar si las instituciones hospitalarias responden a esos límites 
recomendados por la ICNIRP; para así trazar estrategias de protección a las personas que deambulan, trabajan o son atendidos en las mismas. Se puede concluir, observando los resultados obtenidos, que la contaminación electromagnética está presente independientemente de las condiciones de trabajo. Que los valores de radiación que llegan a las personas en una misma área pueden ser diferentes, pues esta depende de si están en movimiento o ubicados en un mismo lugar y del tiempo en que se encuentren en él. (Carvajal et al., 2018).

Las radiaciones electromagnéticas conllevan el peligro de "efectos biológicos" que pueden desencadenar en "efectos adversos" para la salud. Es importante comprender la diferencia entre estos tipos de efectos al estresor electromagnético. Un "efecto biológico" ocurre cuando la exposición produce un cambio en las condiciones fisiológicas detectable en un sistema biológico y un "efecto adverso" ocurre cuando el efecto biológico sobrepasa el límite normal de variabilidad fisiológica del organismo, presentando dificultad de adaptación con detrimento del estado de salud. Algunos "efectos biológicos" pueden ser inocuos, como, por ejemplo, el incremento del flujo sanguíneo en la piel, como respuesta a un ligero calentamiento del cuerpo por la radiación solar, ventajosos como la ayuda en la producción de vitamina D o adversos como el cáncer de piel. (Pérez y Miranda, 2016).

\subsection{Aloe vera como descontaminante electromagnético}

Los extractos vegetales de caléndula, saúco, manzanilla tienen la propiedad de reducir el eritema solar (debido a la acción de moléculas activas como el alfabisabolol procedente del extracto de manzanilla), regaliz y el gel de Aloe vera. Los extractos vegetales son ricos en flavonoides y otras sustancias de acción antiirritante y antiinflamatoria. Actualmente ya está demostrado que el dióxido de titanio en nanopartículas, presentes en cosméticos y bloqueadores solares, generan radicales libres y pueden ocasionar daños al ADN de las células de la piel, provocando desde una simple inflamación de tejidos hasta tumores. El Aloe Barbadensis Miller pertenece al género de aloe herbácea, arbustiva, suculentas xerófilas perennes. De acuerdo con el USDA Aloe vera pertenece a la familia de las liliáceas. El gel de aloe vera mostró muchas actividades fisiológicas y biológicas tales 
como la capacidad de curación de quemaduras en la piel, lesiones cutáneas, acné, la psoriasis, la anemia, anticáncer, el agente anti-viral, protector UV efecto profiláctico contra las radiaciones nucleares accidentales, agente antiinflamatorio, analgésico, antioxidante y también puede ser utilizado como pesticida natural. (Cortes et al., 2020).

\section{Materiales y métodos}

Se realizó una búsqueda de referencias bibliográficas en bases de datos como LILACS, SciELO, PubMed, Scopus, ScienceDirect y Web of Science, empleando descriptores como "Aloe vera", "Contaminación electromagnética", "ultravioleta", así mismo se desarrolló un estudio sistemático de artículos científicos publicados en revistas científicas como Guillermo de Ockham, Dilemas Contemporáneos, Airalia, entre otras, así como también de repositorios universitarios como el de la Universidad Católica de Santiago de Guayaquil, Universidad Tecnológica de la Habana, entre otras, desarrollando el estado de arte en sus fases heurística (mediante la recopilación de artículos científicos, tesis) y hermenéutica (mediante la lectura y el análisis secuencial de la información secundaria)

\section{Resultados}

Las radiaciones electromagnéticas (REM) que se encuentran entre $30 \mathrm{kHz}$ y $300 \mathrm{MHz}$ y las microondas entre $300 \mathrm{MHz}$ y $300 \mathrm{GHz}$, incitan a vibraciones moleculares y producen calor, por lo que se emplea en la industria médica y también uso doméstico, pudiendo producirse quemaduras en la piel humana, donde los órganos más afectados por estas REM son los de poca irrigación como el cristalino y el humor vítreo del ojo, los órganos parenquimatosos y otros como ganglios linfáticos, hígado, páncreas, las gónadas y los órganos huecos como el estómago, la vejiga y la vesícula biliar, teniendo ya evidencia de las consecuencias biológicas de los campos electromagnéticos (CEM), en base al análisis de los estudios epidemiológicos (Pérez et al., 2017), y es que, las consecuencias de las REM en la salud humana no sólo dependen de su intensidad sino también de su frecuencia y energía, siendo las principales fuentes de CEM la red de suministro eléctrico, los aparatos eléctricos y las pantallas de computadora; mientras que, los sistemas de seguridad son las principales fuentes de campos de radiofrecuencia, conjuntamente con la radio, la televisión, las antenas de radares, los teléfonos celulares y los hornos de microondas. 
Los CEM inducen corrientes en el organismo que, dependiendo de su frecuencia y amplitud, pueden ocasionar varios efectos como calentamiento y sacudidas eléctricas; precisamente por ello, si un piloto de avión no mantiene abierta la trompa de Eustaquio mediante movimientos de deglución, o si tiene catarro que determinó la obstrucción de dicho conducto se producirá la ruptura del tímpano, en caso de que el avión caiga en picada a una velocidad vertiginosa, por ello los pilotos evitan volar cuando tienen un resfrío, y si lo hacen, procuran que el ascenso y el descenso sean muy graduales para que no se establezcan diferencias bruscas entre la presión del oído medio y la del oído externo (Catalán, 2020).

\section{Discusión}

De acuerdo a Rodríguez (2006), la actividad biológica del Aloe vera se ha complementado a los carbohidratos (polisacáridos), que está compuesta aproximadamente por el 20 \% de los sólidos totales de las hojas de esta planta, incluso se tiene comprobado que unas 20 proteínas de distintas clases, asociadas con el polisacárido, contribuyen a la actividad farmacológica en la estimulación de la proliferación celular y otras posibles actividades tales como la antinflamatoria y la antiulcerativa, en concordancia con Cortes et al. (2020) quienes aseveran que el uso de la sábila disminuye de alguna manera los efectos nocivos para la salud humana, mediante el proceso de la fotosíntesis, gracias a su gel disponible que se constituye en dos fases: un sistema coloidal de fase continua o sólida y la fase dispersa o líquida, extractos vegetales ricos en flavonoides y otras sustancias de acción antiirritantes y antiinflamatorias.

\section{Conclusiones}

Se logró una evaluación objetiva de las evidencias científicas acerca de los potenciales efectos de los campos electromagnéticos sobre la salud, donde las radiaciones entre $30 \mathrm{kHz}$ y $300 \mathrm{MHz}$ y las microondas entre $300 \mathrm{MHz}$ y $300 \mathrm{GHz}$, provocan vibraciones o alteraciones moleculares, las cuales son producto de un análisis y revisión de estudios epidemiológicos. 
Se encontró una alternativa para mitigar las ondas electromagnéticas, con el uso del gel de aloe vera, en las cuales mostró muchas actividades fisiológicas y biológicas tales como la capacidad de curación de quemaduras en la piel, lesiones cutáneas, acné, la psoriasis, la anemia, anticáncer, el agente anti-viral, protector UV efecto profiláctico contra las radiaciones nucleares accidentales, agente antiinflamatorio, analgésico, antioxidante.

Todavía no entra en aplicación la importancia de diseñar políticas públicas que informen, regulen y limiten la exposición a campos electromagnéticos, por lo que los efectos: riesgo de cáncer, estrés celular, incremento de radicales libres perjudiciales, daños genéticos, cambios estructurales y funcionales en el sistema reproductivo, déficit en la memoria y el aprendizaje, desórdenes neurológicos e impactos negativos en el bienestar, que perjudica a todos los seres vivos.

El daño ambiental constituye un nuevo supuesto de responsabilidad civil, que presenta características jurídicas y procesales aplicables exclusivamente a los derechos ambientales, el actual sistema de responsabilidad civil regulada en el Código Civil, Ley General del Ambiente y Código Procesal Civil, presentan dificultades respecto a la actividad probatoria por daños ambientales.

\section{Referencias}

Carvajal, J. Pérez, I, y Castro. M. (2018). Análisis del Campo Magnético en una Institución Hospitalaria. Energética, 39 (3), 176-185.

Catalán, C. (2020). Enredados en las Ondas del 5G. Recuperado de https://navarracapital.es/enredados-en-las-ondas-del-5g/

Cortes, I., Rodríguez, F., Olalla, J., López, A., Jiménez, A., García, L. y Camacho, B. (2020). Estudios de Biocompatibilidad de Compósito en Gel de Aloe vera y Nanopartículas de TiO2 para uso Dérmico. En E. San Martin Martinez, MA Ramírez Salinas(Eds.). Avances de investigación en Nanociencias, Micro y Nanotecnologías. Barcelona, España: Omnia Science. 133-152. 
Leal, J. (2019). Efectos físicos de la contaminación atmosférica percibidos de manera inconsciente por la ciudadanía, en el área metropolitana de la ciudad de Monterrey, Nuevo León, México. Rev. Salud Pública, 21(4), 1-7.

Ledesma, P. (2019). Guía de la Contaminación Electromagnética y la Salud. Asociación Tarifa Contra el Radar. Tarifa, España. https://n9.cl/f9ekz

Pérez, L. Rodríguez, G. Valdés, Y. Fuentes, R. (2017). El Veneno del Siglo XXI. Rev. Panorama Cuba y Salud, Escuela Latinoamericana de Medicina. Cuba, 12(1 Esp),13-15.

Pérez, J. y Miranda, R. (2016). Radiaciones electromagnéticas y salud en la investigación médica. Med Mil, 39 (1), 180.

Rodríguez, I. (2006). Beneficios del Aloe Vera I. (sábila) en las afecciones de la piel. Rev Cubana Enfermer, 22(3), 1-10.

Rolín, J, Kolodziej, F y Cruz, R. (2014). Contaminación Electromagnética Generada por antenas de Telefonía Móvil. Revista Científico UNAM de la Facultad de Ingeniería OBERA- Argentina.

Sampedro, C. Palma, D. Tinitana, D y Barberán, J. (2020). Contaminación electromagnética por dispositivos de comunicación inalámbrica y sus efectos en la salud. Revista Dilemas Contemporáneos. Educación, Política y Valores, 8(1),157.

Tutivén, P. (2015). Los Efectos de las Radiaciones Electromagnéticas de Radiofrecuencia en la Salud Humana, Universidad Católica de Santiago de Guayaquil - UCSG-2015. Ecuador. Vargas et al., (2020). La Contaminación Electromagnética. Edt. CECAR. ISBN: 978-958-554774-2 (digital) 